\title{
Adaptive Mobility Target Tracking with Metaheuristic Aided Target Movement Prediction Scheme in Wireless Sensor Network
}

Ramadevi Narasapuram ( $\sim$ ramadevi.narasapuram@gmail.com )

Jawaharlal Nehru Technological University Anantapur https://orcid.org/0000-0001-9363-2805

\section{Subramanyam M V S}

Santhiram Engineering College

\section{Shoba Bindu C}

Jawaharlal Nehru Technological University Anantapur

\section{Research Article}

Keywords: Wireless Sensor Network, Target Tracking, Target Location, Extended Kalman Filter, Optimization.

Posted Date: February 23rd, 2022

DOI: https://doi.org/10.21203/rs.3.rs-1023504/v1

License: (a) (1) This work is licensed under a Creative Commons Attribution 4.0 International License. Read Full License 


\title{
Adaptive Mobility Target Tracking with Metaheuristic Aided Target Movement Prediction Scheme in Wireless Sensor Network
}

\author{
N. Ramadevi ${ }^{1 *}$, M.V.S Subramanyam ${ }^{2}$ and C. Shoba Bindu ${ }^{3}$
}

\begin{abstract}
Mobile Target Tracking is one of the most important applications in Wireless Sensor Networks (WSNs), particularly for surveillance purposes. The tracking accuracy is highly dependent on distance estimation or localization, and so far more works has been done in this aspect. This paper proposes a new energy-saving target tracking scheme with two phases: (i) Mobility Target Tracking and (ii) Target Movement Prediction. At first, the target tracking is attained by Extended Kalman Filter. Following this, the target movement is predicted with the aid of input factors such as Angle of Arrival (AoA) and Received Signal Strength (RSS), thereby the mobile node's optimal movement is predicted. This scenario is considered as the optimization crisis as the prediction of optimal node movement is one of most significant problems in WSN. In order to make the optimal prediction more precise, a new hybrid algorithm named Lion Mutated- Crow Search Algorithm (LM-CS) is introduced. The proposed algorithm combines the concept of Lion Algorithm (LA) and Cuckoo Search algorithm (CS), respectively. To the end, the performance of proposed work is evaluated over other models with respect to convergence analysis, error analysis and so on.
\end{abstract}

Keywords Wireless Sensor Network; Target Tracking; Target Location; Extended Kalman Filter; Optimization.

\footnotetext{
N. Ramadevi

ramadevi.narasapuram@gmail.com

1 Research Scholar, Department of CSE, JNTUA, Ananthapuramu, Andhra Pradesh, INDIA

2 Professor, Department of ECE, Santhiram Engineering College, Nandyal, Andhra Pradesh, INDIA

3 Professor, Department of CSE, JNTUA College of Engineering, Ananthapuramu, Andhra Pradesh, INDIA
} 


\section{Nomenclature}

\begin{tabular}{|l|l|}
\hline Abbreviations & \multicolumn{1}{|c|}{ Descriptions } \\
\hline WSN & Wireless sensor network \\
\hline GPS & Global Positioning System \\
\hline RoI & Regions of Interest \\
\hline MSN & Pobile Sensor Network \\
\hline PSO & H-Best PSO \\
\hline HPSO & Line of Sight \\
\hline LoS & Two-Dimensional Localization \\
\hline TDL & Spatial Localization Module \\
\hline SLM & Received Signal Strength \\
\hline RSS & Temporal Localization Module \\
\hline TLM & $\begin{array}{l}\text { Optimal Priority based Trajectory } \\
\text { with Energy Constraint }\end{array}$ \\
\hline OPTEC & Mixed Integer Linear Programming \\
\hline MILP & $\begin{array}{l}\text { Path Planning method for Multiple } \\
\text { Mobile Anchor Nodes }\end{array}$ \\
\hline PP-MMAN Algorithm \\
\hline CAP & $\begin{array}{l}\text { Compensation } \\
\text { Positioning }\end{array}$ \\
\hline IoT & Internet of Things \\
\hline CH & Cluster Head \\
\hline NSPS & Naïve Shortest Path Selection \\
\hline RARE-Area & Reduced Area Reporting-Area \\
\hline EATT & Energy Aware Target Tracking \\
\hline
\end{tabular}

\section{Introduction}

WSN [9-12] is the most promising area due to its capability of monitoring the physical world through a lot of inexpensive and small sensors. Among the application in WSN, some of the most important and critical applications are location awareness, disaster response, geographic routing, vehicle tracking, and environment surveillance, respectively. GPS is the widely used tactic for information gaining in WSNs [13-15]. Though, GPS suffers from many issues under various situations: line shortage of sight to multiple satellites. Further, the equipping or target with the GPA is unfeasible and undesirable as it deals with high hardware cost. Hence, more researchers were under the development of localization models, yet they limits with the static target.

On the other hand, it seems to be the challenge if the position of mobile targets get changed regularly. Some of the challenges in mobility that needs to rectify involves coverage, connectivity and energy consumption. One of the most important issues over the mobile 
WSNs is the precise localization of mobile devices [16-20]. Another issue related to this localization is the coverage problem and is of three categories named barrier coverage, target coverage and area coverage. These three coverage factor are connected to one another. The communication among one another is made by using sensors and is also performed the data filtering and collection to make sure the region monitoring for target coverage.

Similarly, WSN often deals with the crucial applications of target tracking. Target tracking [21-25] and [31] comprised of trajectory detection for object movement, data filtering, routing and gathering via sensors monitoring, conducting intruder detection, and control in RoI. Further, this process contains two major phases like tracking and indexing. Tracking is uninterruptedly progressed via the monitoring process based on the event triggers: intrusion of criminals and adversaries. Further, the main purpose of target tracking is monitoring the target information and satisfying the user needs. Till now, more research works exist in this scenario; still they lags on the better performance in terms of better tracking outcomes.

This framework plans to implement a new energy-saving target tracking scheme with two main phases: (i) Mobility Target Tracking and (ii) Target Movement Prediction. Initially, the Adaptive Extended Kalman Filter is deployed for achieving the target tracking. The target movement prediction is sustained with the help of input factors such as AoA and RSS, which in turns predict the mobile node's optimal movement. For this optimal prediction of target movement, this paper introduces a new hybrid algorithm named LM-CS, which hybrids the concept of LA and CS. The evaluation on performance is made via various analyses and thus proves the superiority of implemented model.

The paper is organized as per as follows: Section 2 details the literature review of the overall contributed papers. Section 3 specifies the system model of proposed target movement prediction in wireless sensor network. Section 4 depicts the working strategy of proposed model. Section 5 explains the proposed hybrid algorithm for estimating the target by combining the Lion and Cuckoo search algorithm. Section 6 delineates the results and their discussions. Section 7 terminates the paper.

\section{Literature Survey}

\subsection{Related Works}

In 2018, Singh et al. [1] has introduced the localization of target nodes with moving single anchor node by proposing CI based application of PSO and HPSO. The Hilbert trajectory was 
followed by this moving anchor node. The actualization of proposed algorithms was made that meant for distributed, range-based, isotropic, non-collaborative WSNs. The reference node was assigned only by a single moving anchor node for localizing the target nodes within the whole network. The challenge of LoS in the proposed algorithms was reduced by means of virtual anchor node's projection.

In 2016, Sun et al. [2] has introduced a new TDL approach using compressive sensing for mobile targets. This was involved with two constraints: (i) SLM that initially gains localization by developing the RSS vector's sparse nature in space domain at sampling times, and (ii) TLM which gains localization at entire times by developing within the time domain. Moreover, for conducting the linear measurements, two practicable measurement matrices were designed. The simulation experiment has shown the superiority of the proposed localization model.

In 2019, Kouroshnezhad et al. [3] has proposed a new mobile anchor trajectory planning model named OPTEC. The MILP optimization technique has been used in the implemented structure to plan the optimal route for deployed sensors using the availability of location uncertainty. Various essential computation metrics were explained for comparing the static mobile anchor trajectory plans. Further, this paper has deployed the static sensors that use a range-free localization method. The experimental outcomes have revealed the betterment of implemented work than the conventional models.

In 2019, Sun et al. [4] has implemented a novel PP-MMAN for enhancing the location efficacy of the node by means of limiting the moving path length and for reducing the anchor node's energy consumption by avoiding the appropriate phenomenon. Additionally, to resolve the issue of few unknown nodes closer to boundary area that doesn't compute its position, a new CAP has been proposed. The implemented PP-MMAN approach has been compared with other models and the outcomes has explained the superior performance with less energy and increased location efficiency.

In 2018, Kuo et al. [5] has presented an adaptive approach of trap coverage using a strong area coverage method deployed with mobile sensors of MSNs applications and IoT. Further, this paper has proposed a robust approach of trap coverage that involves the usage of mobile sensors within target tracking and services detection for MSN applications. The simulation outcomes has validated that the developed model has effectively minimized the total count of 
unavailable sensors and the target-missing time. This has also improved the trap coverage maintenance via the mobile sensor's movement in MSNs.

In 2019, Wang et al. [6] has developed an improved Bayesian based least-square algorithm to move the target tracking and localization in WSNs. A set of sub-range probability on the basis of target predictive location was obtained by applying the enhanced Bayesian algorithm. This creates a range joint probability matrix that was updated automatically, while the testbed of WSN is in dormant form. Subsequently, the calculation and normalization of weight of each measurement was made depends on the probability of range matrix. To the end, in accordance to the weighted least-square algorithm, the calculation of target prediction position's corrected value was made. The analysis on this proposed model has revealed the reduced computational burden with better improvement.

In 2018, Lersteau et al. [7] has addressed the challenge on balancing and preserving the residual capabilities of the sensors, for carrying out target tracking missions by utilizing the similar WSN. For this purpose, this research work has proposed a two-step exact approach. Initially, for modelling the mathematical formulation, the processing of input data was made. Secondly, they proposed a hybrid algorithm that combined the column generation algorithm with a GRASP metaheuristic. The overall performance analysis has thus proved the improvement of proposed model over other works.

In 2019, Amir [8] has introduced a target tracking model with minimized energy consumption. Further, this work has proposed a prediction method by means of combining the active modes and Medium Access Control layer for tracking the target when the remaining nodes were in sleep mode. In the network, the nodes were classified as target monitoring, target detection, and off modes. The other available nodes sense the target movement and alter their situation as detection mode. As long as the target was visible, the target tracking process has been continued via representative nodes. The experimental analysis has thus revealed the improvement of the proposed work over the conventional models. 
Table 1 Impact of localization and tracking problem in Mobile Wireless Sensor Network

\begin{tabular}{|c|c|c|c|}
\hline $\begin{array}{l}\text { Author } \\
\text { [citation] }\end{array}$ & Methodology & Features & Challenges \\
\hline Singh et al. [1] & PSO and HPSO & $\begin{array}{l}\text { - } \quad \text { Fast convergence rate } \\
\text { - Can be implemented in } \\
\text { logistics and military }\end{array}$ & $\begin{array}{l}\text { - Needs hybrid algorithms } \\
\text { to be propose in future } \\
\text { - Needs enhancement in } \\
\text { accuracy }\end{array}$ \\
\hline Sun et al. [2] & TDL & $\begin{array}{l}\text { - Significantly minimizes } \\
\text { the spatial and temporal } \\
\text { measurement numbers } \\
\text { - } \quad \text { Better effectiveness and } \\
\text { robustness }\end{array}$ & $\begin{array}{l}\text { - Communication cost is } \\
\text { high }\end{array}$ \\
\hline $\begin{array}{l}\text { Kouroshnezhad } \\
\text { et al. }[3]\end{array}$ & MILP & $\begin{array}{l}\text { - } \text { Better determination of } \\
\text { beacon point } \\
\text { - Improved trajectory } \\
\text { efficiency, localization } \\
\text { accuracy and sensor } \\
\text { nodes lifetime }\end{array}$ & $\begin{array}{l}\text { - } \text { Needs solution to mobile } \\
\text { anchor path planning } \\
\text { optimization problem }\end{array}$ \\
\hline Sun et al. [4] & PP-MMAN & 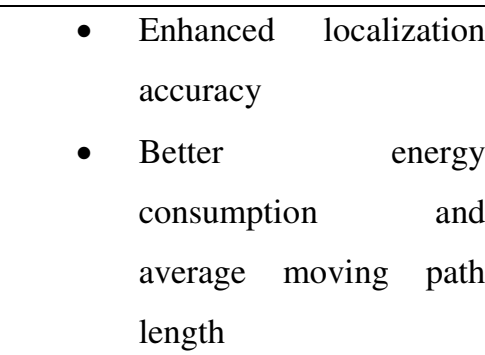 & $\begin{array}{l}\text { - Designing in dynamic } \\
\text { path planning needs } \\
\text { further study } \\
\text { - Needs intelligent model to } \\
\text { avoid the obstacles }\end{array}$ \\
\hline Kuo et al. [5] & $\begin{array}{ll}\mathrm{Nr}_{\mathrm{s}} & \mathrm{Trap} \\
\text { Coverage } & \end{array}$ & $\begin{array}{l}\text { - Minimizes the target } \\
\text { missing time } \\
\text { - Reduces the count of } \\
\text { unavailable sensors }\end{array}$ & $\begin{array}{l}\text { It is hard to } \\
\text { computationally calculate } \\
\text { the long distance }\end{array}$ \\
\hline Wang et al. [6] & $\begin{array}{l}\text { Improved } \\
\text { Bayesian } \\
\text { Enhanced Least- } \\
\text { Squares } \\
\text { Algorithm }\end{array}$ & $\begin{array}{l}\text { - Reduced the localization } \\
\text { RMSE } \\
\text { - Minimized } \\
\text { computational time } \\
\text { consumption }\end{array}$ & $\begin{array}{llr}\text { - } & \text { Needs } \\
\text { improvement } & \text { in } \\
\text { optimization performance }\end{array}$ \\
\hline $\begin{array}{l}\text { Lersteau et al. } \\
\text { [7] }\end{array}$ & $\begin{array}{l}\text { Column } \\
\text { generation } \\
\text { algorithm }\end{array}$ & $\begin{array}{l}\text { - Significantly reduces the } \\
\text { CPU time }\end{array}$ & $\begin{array}{l}\text { - Increased communication } \\
\text { cost } \\
\text { - Energy can be drained } \\
\text { easily }\end{array}$ \\
\hline
\end{tabular}




\begin{tabular}{|l|l|l|l|l|}
\hline Amir [8] & EATT & $\begin{array}{l}\text { Better energy } \\
\text { consumption }\end{array}$ & $\begin{array}{l}\text { Optimization concept } \\
\text { needs to be evolved }\end{array}$ \\
& & High throughput & $\begin{array}{l}\text { Delay and time slots have } \\
\text { to be presented in } \\
\end{array}$ & \\
& & simulation
\end{tabular}

\subsection{Review}

Table 1 specifies the impact of localization and tracking problem in Mobile Wireless Sensor Network. From the above review on the papers, it was clear that the localization and tracking are the two main challenges that need to be considered in WSN for effective transmission. Convergence and connectivity are the two major factors that existed in this localization and tracking. Hence, more models are evolved in the existed works to enhance these issues on localization and tracking, still so many issues are there to solve. This explains the features and challenges of the method that existed in the literature as follows: PSO and HPSO [1] has fast convergence rate and can be implemented in logistics and military. However, needs hybrid algorithms and further needs enhancement in accuracy. TDL [2] has significantly minimizes the spatial and temporal measurement numbers and pose better effectiveness and robustness, but the communication cost is high. MILP [3] achieves better determination of beacon point and has improved trajectory efficiency, localization accuracy and sensor nodes lifetime. The main issues related to this methodology is, it needs solution to mobile anchor path planning optimization problem. PP-MMAN [4] pose enhanced localization accuracy and better energy consumption and average moving path length. Though designing in dynamic path planning needs further study and needs intelligent model to avoid the obstacles. Nrs Trap Coverage [5] minimizes the target missing time and reduces the count of unavailable sensors, but, it is hard to computationally calculate the long distance. Improved Bayesian Enhanced Least-Squares Algorithm [6] has reduced localization RMSE and computational time consumption. However, the main drawback is it further needs improvement in optimization performance. Column generation algorithm [7] significantly reduces the CPU time, yet pose increased communication cost and the energy can be drained easily. EATT [8] has better energy consumption and high throughput. Optimization concept needs to be evolved and, delay and time slots have to be presented in simulation are the two main issues of this method. 


\section{Proposed Target Movement Prediction in Wireless Sensor Network: System Model}

\subsection{System Model}

Consider $N$ static sensors $s_{i}: i=1,2, \ldots, N$ that are evenly distributed in the sensing area. The location of each sensor is defined under $\left(x_{i}, y_{i}\right)$ coordinates. Similarly, the target $T$ is assumed to be moving along a certain path. The location of $T$ is defined as $\left(x_{T}, y_{T}\right)$. Fig. 1 depicts the diagrammatic representation of target and sensors in a network.

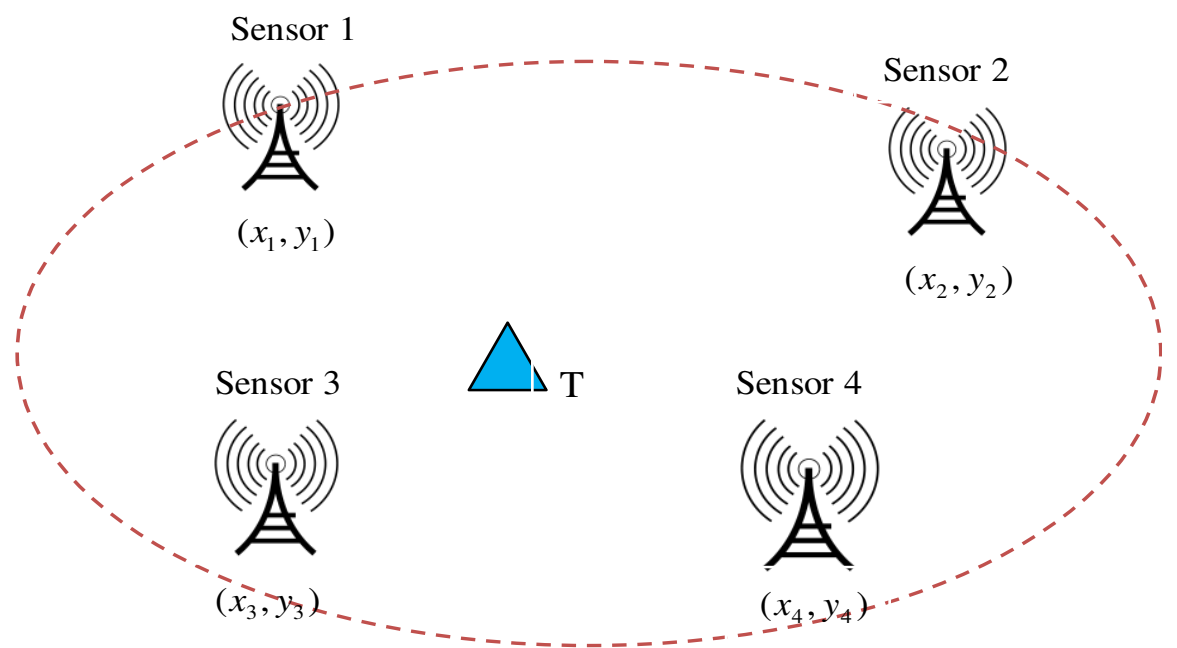

Fig. 1 Diagrammatic representation of target and sensors in a network

The problem defined in this work is the minimization of tracking error. The tracking error indicates the average tracking error among the real location $\left(x_{T}, y_{T}\right)$ and estimated location $\left(\hat{x}_{T}, \hat{y}_{T}\right)$ when the target is being tracked for certain time interval $t$. This evaluation is given in Eq. (1), where $\left(x_{T}, y_{T}\right)$ is unknown.

$$
\arg \min \underset{\left(\hat{x}_{T}, \hat{y}_{T}\right)}{\operatorname{er}}=\frac{1}{T} \sum_{T}^{i=1} \sqrt{\left(\hat{x}_{T}-x_{T}\right)^{2}-\left(\hat{y}_{T}-y_{T}\right)^{2}}
$$

\subsection{Use of Static Sensor}

In this work, the sensors sense the location of the target when it obtains the range from the sensor with respect to RSS and AoA. However the sensors are incapable of estimating 
bearing directly, as because of the additive noise with the reflected signal. This paper introduces an Adaptive Extended Kalman filter based localization algorithm for predicting the target. Moreover, the proposed algorithm determines the hybrid function that involves AoA, RSS and distance of the signal.

\subsection{Derivation of RSS}

The receiving noisy RSS that received from target by each static sensor is given in Eq. (2). In Eq. (3), $d_{i}$ portrays the distance between the target and sensor and the mathematical expression of this is given in Eq. (4).

$$
\begin{gathered}
\overline{R S S}_{i}=P_{i}+n \\
P_{i}=P_{o}-10 * \beta^{*} \log _{10}\left(\frac{d_{i}}{d_{o}}\right) \\
d_{i}=\operatorname{dist}\left(\left(x_{i}, y_{i}\right),\left(x_{T}, y_{T}\right)\right)
\end{gathered}
$$

Here, $i=1,2, \ldots, 4$ as we have assumed four static sensors. From the given equation, the distance $\bar{d}_{i}$ should be calculated as per Eq. (5). From each $\overline{R S S}_{i}$, the corresponding $\bar{d}_{i}: i=1,2, \ldots, 4$ is evaluated.

$$
\begin{gathered}
\bar{d}_{i}=d_{0} 10^{x} \\
x=\frac{P_{o}-R S S}{10 \beta}
\end{gathered}
$$

\subsection{Derivation of AoA}

Similarly, the noisy AoA from target by each static sensor is determined $\bar{a}_{i}: i=1,2, \ldots, 4$, where $\bar{a}$ is the original AoA evaluated on distance between the original location and static sensor.

\section{Working Strategy of Proposed Model}

Fig. 2 shows the systematic working strategy of proposed tracking model. From the received static sensor data, the historical movements of the target get trained in EKF. With the target movement characteristics (distance), the EKF will predict the approximate location $\left(x_{T}, y_{T}\right)$ of the target. 


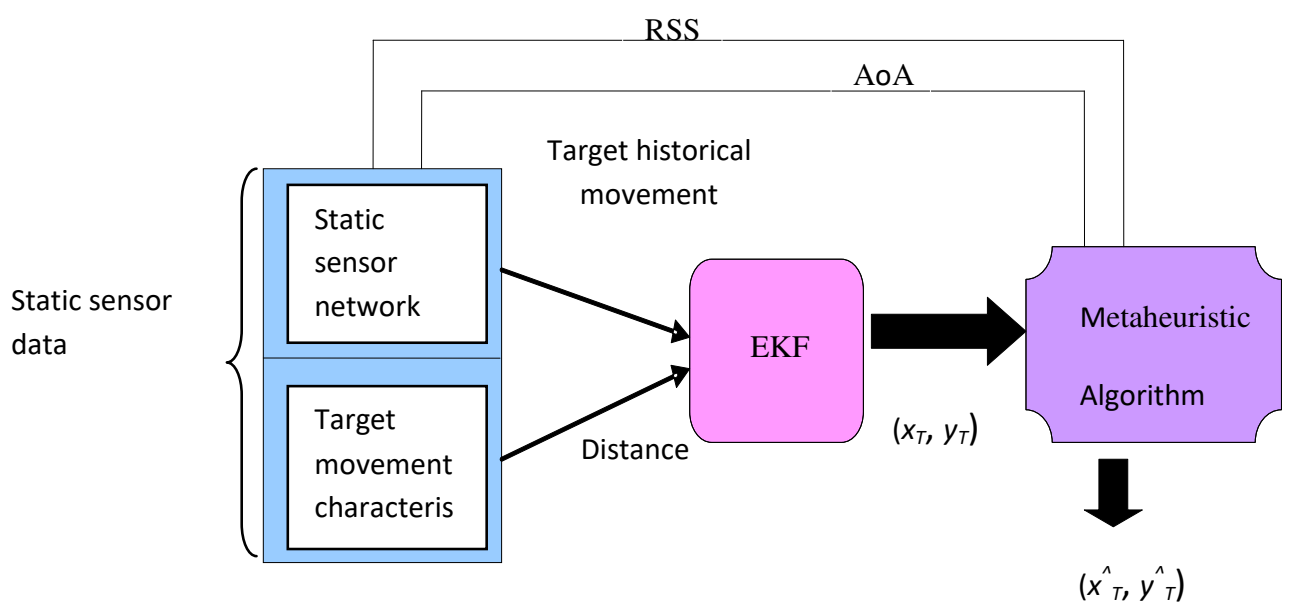

Fig. 2 Architecture of Proposed Model

\subsection{Extended Kalman Filter}

The model's state vector is defined as per Eq. (7).

$$
w=\left[f_{0} \dot{f}_{0} \mathrm{~g}_{0} \dot{\mathrm{g}}_{0} h_{0} \dot{h}_{0} \quad \varphi_{0} \dot{\varphi}_{0} \vartheta_{0} \dot{\vartheta}_{0} \psi_{0} \dot{\psi}_{0}\right]^{T}
$$

Consider the objective velocity as constant over a sample time interval $T$. Assume the corresponding dynamic modelling error as $\gamma_{k}$. The model on discrete time dynamic is given as per Eq. (8), in which, $A$ is the state transition matrix and is constant.

$$
w_{k}=A w_{k-1}+\gamma_{k}
$$

The kalman filter output are selected as the normalized coordinate vector of $\bar{m}$ feature point of the camera's image plane is expressed using Eq. (9), in which, the observation noise vectors for the components $u$ and $v$ of the normalized image plane is portrayed as $v_{k}^{u}$ and $v_{k}^{v}$.

$$
\begin{aligned}
& \zeta_{k}^{u}=b^{u}\left(w_{k}\right)+v_{k}^{u} \\
& \zeta_{k}^{v}=b^{v}\left(w_{k}\right)+v_{k}^{v}
\end{aligned}
$$

Further, $b^{u}\left(w_{k}\right)$ and $b^{v}\left(w_{k}\right)$ vector functions are depicted as per Eq. (10).

$$
\begin{aligned}
& b^{u}\left(w_{k}\right)=\left[\frac{{ }^{c} f_{1}}{{ }^{c} h_{1}} \ldots \frac{{ }^{c} f_{\hat{m}}}{{ }^{c} h_{\hat{m}}}\right]_{k}^{T} \\
& b^{v}\left(w_{k}\right)=\left[\frac{{ }^{c} g_{1}}{{ }^{c} h_{1}} \ldots \cdot \frac{{ }^{c} g_{\hat{m}}}{{ }^{c} h_{\hat{m}}}\right]_{k}^{T}
\end{aligned}
$$


The disturbance quantity components $\gamma_{k}, v_{k}^{u}$ and $v_{k}^{v}$ are assumed as non-stationary, independent, white noise, Gaussian sequences having statistical properties.

$$
\begin{aligned}
& E\left[\left(\gamma_{k}-q_{k}\right)\left(\gamma_{l}-q_{l}\right)^{T}\right]=Q_{k} \delta_{k l} \\
& E\left[\left(v_{k}^{u}-r_{k}^{u}\right)\left(v_{l}^{u}-r_{l}^{u}\right)^{T}\right]=R_{k}^{u} \delta_{k l} \\
& E\left[\left(v_{k}^{v}-r_{k}^{v}\right)\left(v_{l}^{v}-r_{l}^{v}\right)^{T}\right]=R_{k}^{v} \delta_{k l}
\end{aligned}
$$

In this, the statistical mean operator adapted to the matrix or vector component is stated as $E[$.$] and the Kroneker symbol is expressed as \delta$. As the output method in the system state is nonlinear, the output equations need to be linearized over the present state estimate at every sample time. This directs to the new term named EKF.

On the other hand, the output position from EKF is varied with respect to certain variation $( \pm 4, \pm 6, \pm 8, \pm 10, \pm 12$ ) for estimating the actual target by the proposed hybrid algorithm. Thereby, the estimated target is denoted as $\left(\hat{x}_{T}, \hat{y}_{T}\right)$.

\subsection{Solution Encoding and Objective Function}

The solution that is given as the input to the proposed mobility tracking model is illustrated in Fig. 3. The overall objective function or the fitness of proposed meta-heuristic enabled target prediction model is given in Eq. (12), where $e_{1}$ indicates the objective function 1 under RSS, which is given in Eq. (13) and $e_{2}$ indicates the objective function 2 under AoA and it is given in Eq. (14).

$$
\begin{aligned}
& F=\min \left(\frac{e_{1} e_{2}}{e_{1}+e_{2}}\right) \\
& e_{1}=\sqrt{\sum_{i=1}^{N}\left(\hat{d}_{i}-\bar{d}_{i}\right)^{2}} \\
& e_{2}=\sqrt{\sum_{i=1}^{N}\left(\hat{a}_{i}-\bar{a}_{i}\right)^{2}}
\end{aligned}
$$

In Eq. (13) $\hat{d}_{1}, \hat{d}_{2}, \ldots, \hat{d}_{4}$ indicates the distance between the estimated location from EKF/proposed and static sensor 1,2, 3 and 4.

$$
\hat{d}_{i}=\left(\left(x_{i}, y_{i}\right),\left(\hat{x}_{i}, \hat{y}_{i}\right)\right) ; i=1,2, . ., 4
$$

Similarly in Eq. (14), $\hat{a}_{1}, \hat{a}_{2}, \ldots, \hat{a}_{4}$ indicates the estimated AoA, which is the distance between the estimated EKF/proposed and static sensor 1, 2, 3 and 4. 


$$
\left(x_{T}, y_{T}\right)
$$

Fig. 3 Solution Encoding

\section{Proposed Hybrid Algorithm for Estimating the Target}

\subsection{Lion Algorithm}

LA [26] is inspired based on the concept of Lion's social behaviour and is a well-known meta-heuristic algorithm. Main processing stages are: Fertility evaluation, Territorial takeover, Mating, Pride generation, Territorial defence, and Termination.

Take on the objective function as Eq. (16). Here the real numbers are represented as $\mathfrak{R}$, the continuous unimodal or multimodal function for which the size of the solution space $\mathfrak{R}^{n}$ is given as $\mathfrak{R}^{n}, m_{i}: i=1,2, \ldots, n$, is depicted as the $i^{\text {th }}$ solution variable and the solution space dimension is considered as $n$, the $i^{\text {th }}$ solution variable's maximum and minimum limits is referred to $m_{i}^{\max }$ and $m_{i}^{\min }$, respectively. The solution space size $f(\bullet)$ is discovered using Eq. (17). On considering this LA model, the optimal/target solution to be achieved is stated as $M^{o p}$ and is given as per Eq. (18). In Eq. (16), the solution vector with $M=\left[m_{1}, m_{2}, . ., m_{n}\right]$ representation is portrayed as $M$.

$$
\begin{aligned}
M^{o p}=\underset{m_{i} \in\left(m_{i}^{\min }, m_{i}^{\max }\right)}{\arg \min } f\left(m_{1}, m_{2}, \ldots ., m_{n}\right) ; \quad n \geq 1 \\
\begin{aligned}
\mathfrak{R}^{n}=\prod_{i=1}^{n}\left(m_{i}^{\min }-m_{i}^{\max }\right) \\
M^{o p}=M: f(M)<f\left(M^{\prime} \mid M^{\prime} \neq M ; m_{i}^{\prime}\right. \\
\left.\in\left(m_{i}^{\min }, m_{i}^{\max }\right)\right)
\end{aligned}
\end{aligned}
$$

Pride generation: The prime part in this generation is the pride initialization and is done by using territorial lion $M^{\text {male }}$, its territorial lioness $M^{\text {female }}$ and a nomadic lion $M^{\text {nomad }}$. Here, the nomadic lion is not the member of this pride group, yet it will be considered. The lion and solution vector presentation are same. $m_{k}^{\text {male }}, m_{k}^{\text {female }}$ and $m_{k}^{\text {nomad }}$ are predicted as the vector element of $M^{\text {male }}, M^{\text {female }}$ and $M^{\text {nomad }}$, and are random integers with minimum and maximum limits while $n>1$, here $k$ is assigned to be in range 1 to $K$. In this, the Lion's length is expressed as $K$ and is evaluated as per Eq. (19). 


$$
K= \begin{cases}n ; & n>1 \\ r ; & \text { otherwise }\end{cases}
$$

In Eq. (19), the integers are viewed as $r$ and $n$, for deciding the length's lion. Besides $n=1$, the binary encoded lion are to be explored and thereby the generation of vector element is done using 0 or 1, offered the constraints that are granted in Eq. (20) and (21) ought to be persuaded.

One among the two conditions of nomadic lion is fed up by the produced $M^{\text {nomad }}$ since two nomadic lions are sought on assaulting the territory. Only on territorial defense, the other nomadic lions will be instigated and at that time the position is remained as NULL and is expressed as $M_{1}^{\text {nomad }}$.

$$
\begin{aligned}
& k\left(m_{k}\right) \in\left(m_{k}^{\min }, m_{k}^{\max }\right) \\
& r \% 2=0
\end{aligned}
$$

where

$$
h\left(m_{k}\right)=\sum_{k=1}^{K} m_{k} 2^{\left(\frac{k}{2}-k\right)}
$$

Fertility Evaluation: On taking the fitness when $M^{\text {male }}, M^{\text {female }}$ gets saturated, they attains one of the local or global optima, where the better solution is obtained. Fertility evaluation has supported on skipping from solution on local optimum. From this, the $M^{\text {male }}$ achieves the laggard position when $f\left(M^{\text {male }}\right)$ is larger than $f^{r e f}, L r$ that is the laggardness rate is elevated by one. $f^{r e f}$ indicates the reference fitness. The evaluation of territorial defense is made if the $L r$ overcomes the maximum limit and are stated as $L r^{\max }$. The sterility rate $S r$ is for the fertility confirmation of $M^{\text {female }}$ and after the crossover process, $S r$ is elevated by one. if $S r>S r^{\max }$ that is the tolerance, the update of $M^{\text {female }}$ is carried out using Eq. (23). While the female update $M^{\text {femalt }}$ is assigned as $M^{\text {female }}$ because of its improvement, the mating process is happened. On the other hand, the update process moves on until $h_{c}$ accomplishes $h_{c}{ }^{\max }$, where $h_{c}$ explains the female generation count. If $M^{\text {female }}$ is not replaced by $M^{\text {female }}$, then it may consider $M^{\text {female }}$ as fertile and can produce better cubs.

$$
m_{k}^{\text {female+ }}= \begin{cases}m_{d}^{\text {female }+} & \text { if } k=d \\ m_{k}^{\text {female }} & \text { otherwise }\end{cases}
$$




$$
\begin{gathered}
m_{d}^{\text {female }+}=\min \left[m_{d}^{\max }, \max \left(m_{d}^{\min }, \nabla_{d}\right)\right] \\
\nabla_{d}=\left[m_{d}^{\text {female }}+\left(0.1 \bar{r}_{2}-0.05\right)\left(m_{d}^{\text {male }}-\bar{r}_{1} m_{d}^{\text {female }}\right)\right]
\end{gathered}
$$

The random integer that generated in the range $[1, \mathrm{~K}]$ is indicated as $d$, the $k^{\text {th }}$ and $d^{\text {th }}$ vector element of $M^{\text {female }}$ is denoted as $m_{k}^{\text {female+ }}$ and $m_{d}^{\text {female+ }}$, respectively. Further, the random integer generated in the range $[0,1]$ is expressed as $\bar{r}_{1}$ and $\bar{r}_{2}$ and the female update position is elicited as $\nabla$.

Mating: In LA's mating process, there exist two primary phases and one supplementary phase. Crossover and mutation are the core two processes within primary steps and the gender clustering is exploited in the supplementary phase. The cubs are produced by $M^{\text {male }}$ and $M^{\text {female }}$ via crossover and mutation process. The cubs are the solution which taken from the parents $M^{\text {male }}$ and $M^{\text {female }}$. The crossover process creates four counts of cubs and the remaining four are obtained by mutation.

Lion operators: The territorial defense can sequence as survival fight, creating nomad coalition and besides the nomad and pride coalition updates. The winner take-all approach is adopted for simplifying the nomad coalition process to identify $M^{e_{-} \text {nomad }}$. If the specification explained in Eq. (26)-(28) is fulfilled, the selection of $M^{e_{-} \text {nomad }}$ takes place.

$$
\begin{gathered}
f\left(M^{e_{-} \text {nomad }}\right)<f\left(M^{\text {mal }}\right) \\
f\left(M^{e_{-} \text {nomad }}\right)<f\left(M^{\text {male_cub }}\right) \\
f\left(M^{e_{-} \text {nomad }}\right)<f\left(M^{\text {female_cub }}\right)
\end{gathered}
$$

Territorial takeover operates the updating of $M^{\text {male }}$ and $M^{\text {female }}$ once after the adulthood attainment by $M^{\text {malecub }}$ and $M^{\text {femalecub }}$. The cubs take upon the maximum age $A_{\max }$.

Termination: This LA model terminates if two statements given in Eq. (29) and (30) gets satisfied.

$$
\begin{gathered}
C_{\text {gen }}>C_{\text {gen }}^{\max } \\
f\left(M^{\text {male }}\right)-f\left(M^{\text {optimal }}\right) \leq E_{\text {trh }}
\end{gathered}
$$

In this, the generation count is explained as $C_{g e n}$, which is primarily taken as zero and then elevated by one as per the process of territorial takeover. The absolute difference is 
delineated as $|\bullet|$ and, the error threshold and the maximum count of generation is specified as $E_{t r h}$ and $C_{g e n}{ }^{\max }$, respectively.

\subsection{Cuckoo Search Algorithm}

CS [27] algorithm is considered as a nature-inspired optimization strategy that developed based on the reproduction of cuckoo bird. When operating with CS algorithm, it is much necessitates connecting the possible solutions with cuckoo eggs. Normally, the fertilized eggs of cuckoos are left inside the other cuckoo's nest, and it expected to be grown by the surrogate parents. Sometimes, the foreign eggs are identified by the cuckoos and in this situation, the cuckoos may abundant their entire nest or eliminates that foreign egg.

The CS algorithm is usually performed depending on three set of rules that are mentioned below:

* Every cuckoo takes on a random nest and lays their egg on that.

* The successive generation will be packed through best nests with higher quality eggs.

* In the view of fixed nest count, the foreign eggs are determined by host cuckoo in the probability of $\varepsilon[0,1]$. In this scenario, there is chance for neglecting the foreign egg or else the cuckoo leave out the nest completely and then it constructs a new nest in a new place.

The evaluation of final rule is made after the replacement of fraction $b_{e}$ of $\hat{n}$ host nests with new random solution or new nests. The solution's quality or fitness and the objective function value are simply proportional to each other. In the view of implementation, each egg in the nest is considered as a solution, and every cuckoo lay only one egg that demonstrates one solution. There is no need for making deference among a nest, an egg, or a cuckoo. The main intension is utilizing the new and potentially fine solution (that is cuckoo egg) to substitute a worst solution within the nest.

In the case of global optimization issues, the CS algorithm is very much efficient as it sustains stability among local and the global random walk. The stability among global and local random walks can be inhibited using a switching parameter $b_{e} \varepsilon[0,1]$. Eq. (31) and (32) defines the local and global random walks, correspondingly.

$$
l_{i}^{t+1}=l_{i}^{t}+\alpha q \otimes J\left(b_{e}-\varepsilon\right) \otimes\left(l_{j}^{t}-l_{k}^{t}\right)
$$




$$
l_{i}^{t+1}=l_{i}^{t}+\alpha \operatorname{Le}(q, \lambda)
$$

where, present position chosen by random permutation is $l_{i}^{t}$ and $l_{k}^{t}$, positive step size scaling factor is $\alpha, l_{i}^{t+1}$ exemplifies the subsequent position, the step size is denoted by $q$, $J$ defines the heavy side function, $b_{e}$ has used to exchange local and global random walks, random number from uniform distribution is $\varepsilon$ and levy distribution is indicated as $\operatorname{Le}(q, \lambda)$. The pseudo code of conventional Cuckoo Search algorithm is given in Algorithm 1.

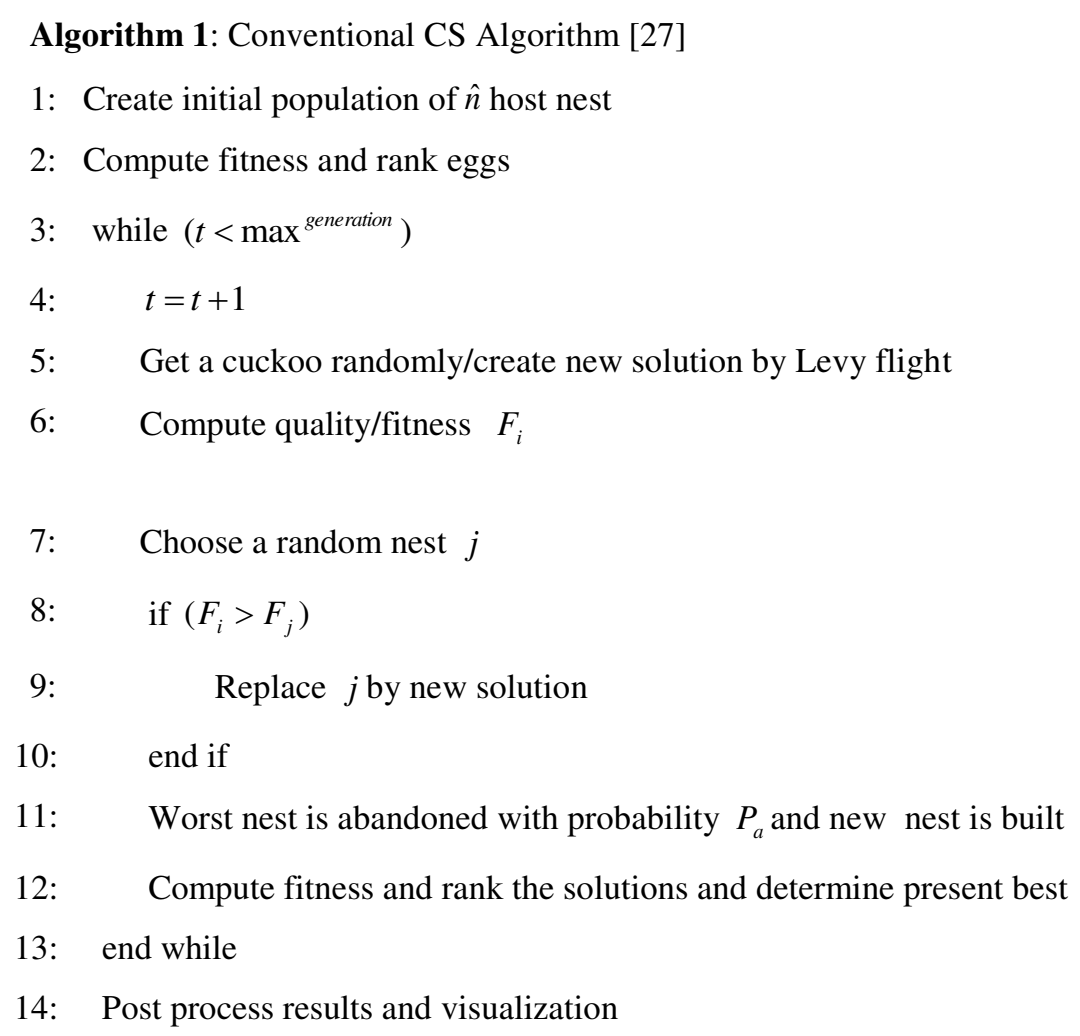

\subsection{Proposed Lion Mutated Cuckoo Search Algorithm}

Even though, the conventional CS and LA models are preferred for solving complex optimization issues, the convergence speed and rate must be increased in many of the multiobjective problems. Hence, this paper made an attempt to introduce a new hybrid algorithm that insists the LA working principle into CS model. The major modification deals in the updating process of solutions.

The proposed algorithm is explained as follows: initially, the population size $\hat{n}$ is fixed to a constant value $10(\hat{n}=10)$. The update process of solutions is based on the condition: for $(\hat{n} \leq 3)$, the update is done as per the cuckoo search update as in Eq. (31). On the condition if 
the host nest population $\hat{n}$ lies among 4 to 6 , the solution gets updated as per the female update equation of LA algorithm in Eq. (23). For $((\hat{n}=7) \&(\hat{n}=8))$, the update process is made as per the mutation process, and on other condition $((\hat{n}=9) \&(\hat{n}=10))$, the update process is evaluated randomly. The pseudo code of proposed LM-CS algorithm is given in Algorithm 2. The flowchart of proposed LS-CS algorithm is illustrated in Fig. 4.

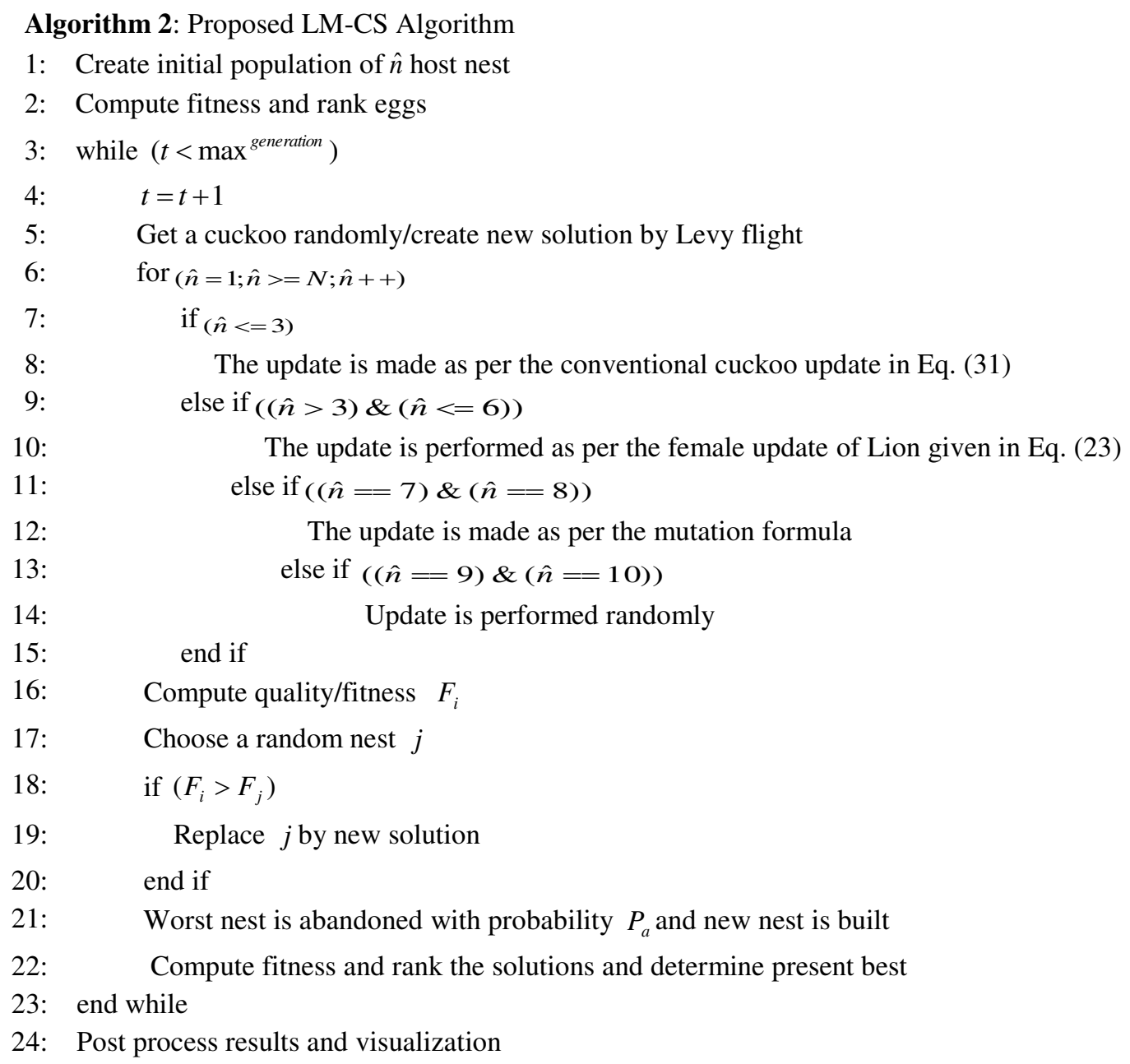




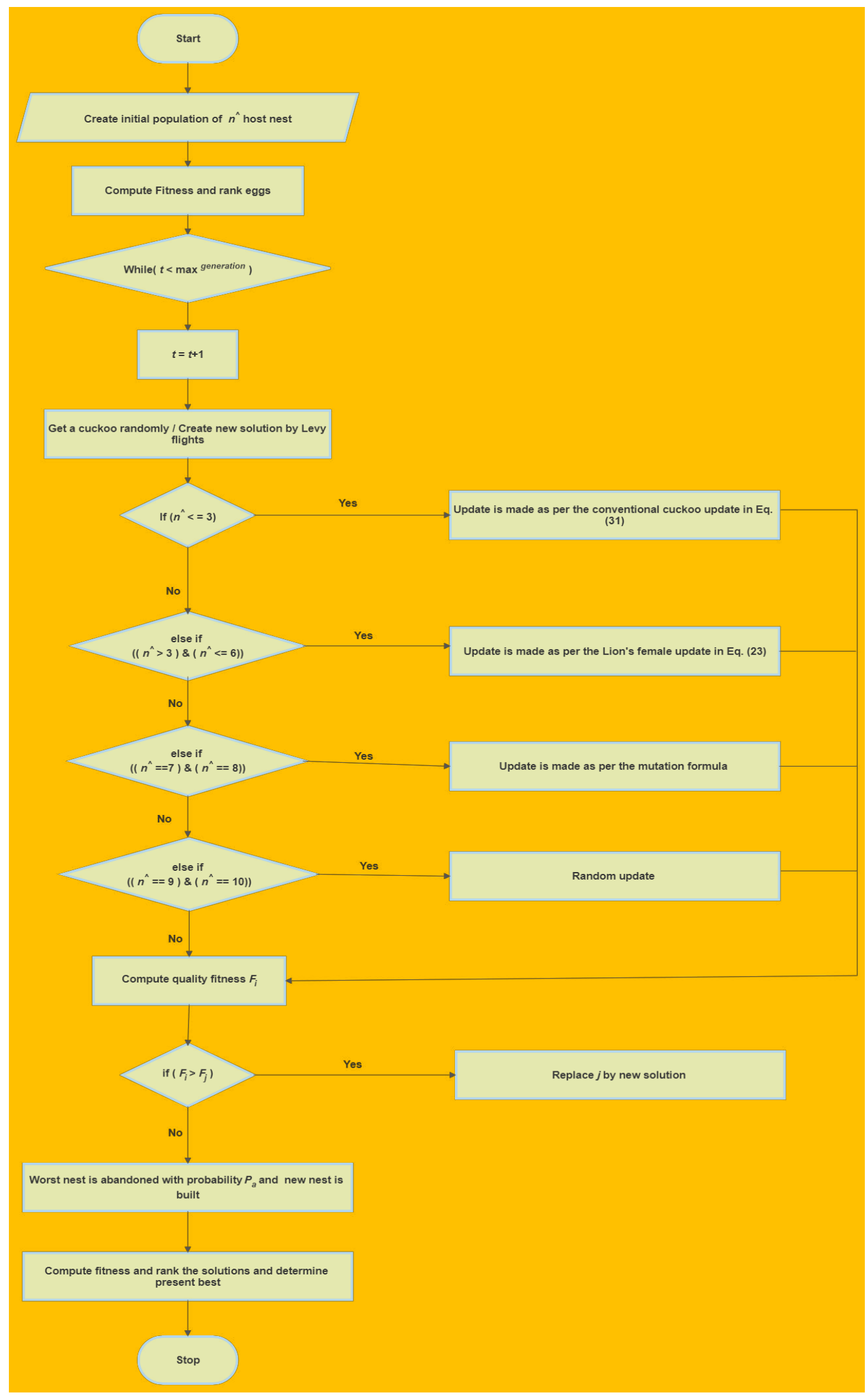

Fig. 4 Flowchart of Proposed LM-CS Algorithm 


\section{Results and Discussions}

\subsection{Simulation setup}

The proposed model was implemented in MATLAB. The performance of the proposed model was compared over the other conventional methods like EHO [28], PSO [29], LA [26], CS [27] and SSA [30] and the results were analyzed. The analysis was made with respect to error measures like MAE, MSE, MASE and RMSE. This error analysis was performed by varying the search space, count of static sensor, noise and reference distance. Further, the convergence analysis and statistical analysis was performed over other conventional methods.

\subsection{Error Analysis by varying Search Space}

Fig. 5 exhibits the error analysis of proposed model over other models. In this section, the analysis is made by varying the search space and number of sensors. The search space is varied as 4, 6, 8, 10 and 12, and the number of sensors are varied as 2, 4, 6, 8 and 10. The error gets decreased as the search space and counts of sensors increases, which shows the improvement of prediction accuracy. From the analysis, it is observed that initially the MAE is high (0.7725) when the search space is 4 and no of sensors are 2 . Then it gradually reduced with increase in search space and count of sensors. It can say that at search space 12 and count of sensors as 10 , the implemented model has achieved a reduced error performance of 0.5432. Similar analysis is done for all other error measures like MSE, MAPE, and RMSE, respectively. Hence, the performance of proposed target prediction model is proved to be better than the conventional models with less error.

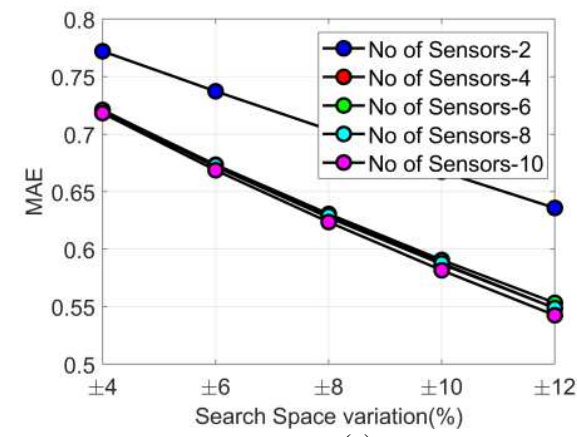

(a)

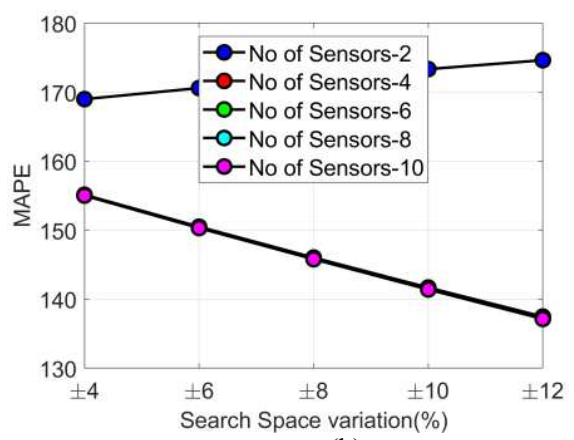

(b) 


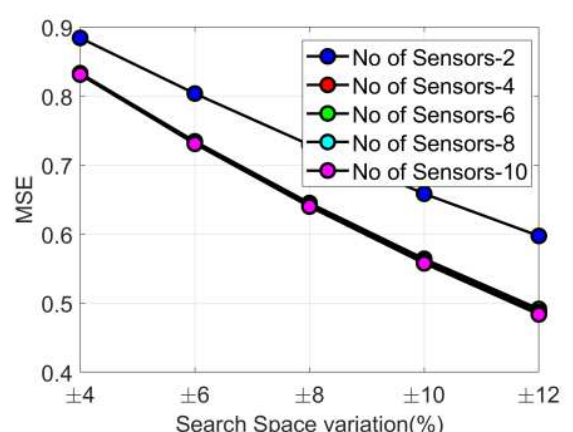

(c)

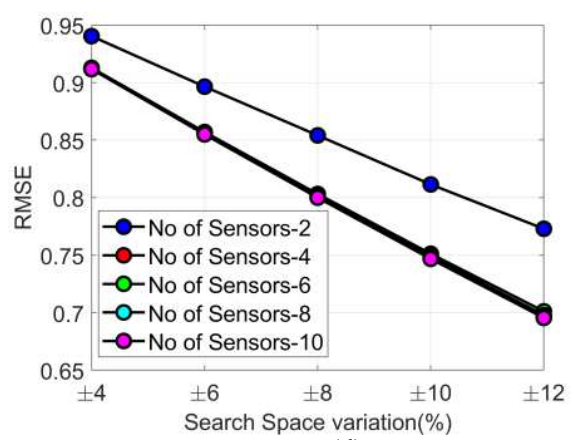

(d)

Fig. 5 Error Analysis of Proposed model by varying the search space and number of sensors (a) MAE (b) MAPE (c) MSE and (d) RMSE

\subsection{Error Analysis by varying Noise}

Fig. 6 explains the error analysis of proposed model over other state-of-the-art models by varying the noise. In fact, as the noise in signal gets increased, the error gets increased to a certain level. From the graphs, it is observed that initially, there obtains minimum error with least signal noise. As the noise increases, error gets increased. However, the proposed model attains minimum error even with high noise. While analysing MAE, the implemented model with noise variance 0.15 has achieved better results with least error, which is $0.45 \%$ and $0.3 \%$ improved than EHO and PSO, respectively. Similarly, for noise variance 0.3, the presented model is $0.32 \%$ and $0.27 \%$ superior to EHO and PSO, respectively. On considering the MSE measure, the proposed model in noise variance 0.3 gained supremacy than conventional models EHO and PSO by $0.18 \%$ and $0.07 \%$, respectively with minimum error. In the view of MAPE measure, the proposed model at noise variance 0.15 is $0.27 \%$ and $0.07 \%$ better from EHO and PSO, respectively. The developed model regarding RMSE measure with noise variance 0.3 is $0.12 \%$ and $0.09 \%$ better than conventional models like EHO and PSO, respectively. Hence the supremacy of proposed model is proved over other models with minimum error. 


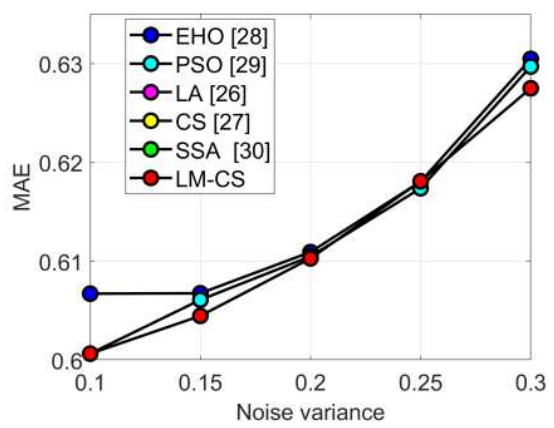

(a)

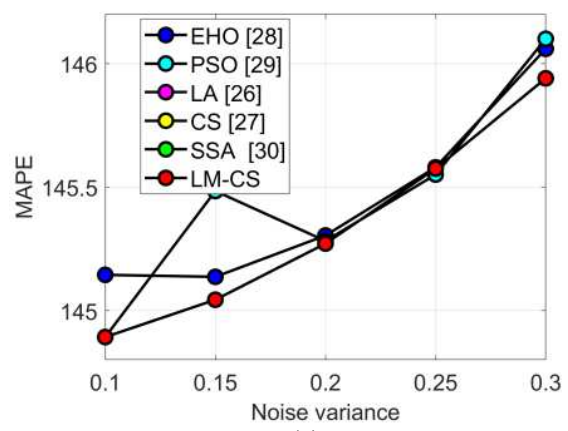

(c)

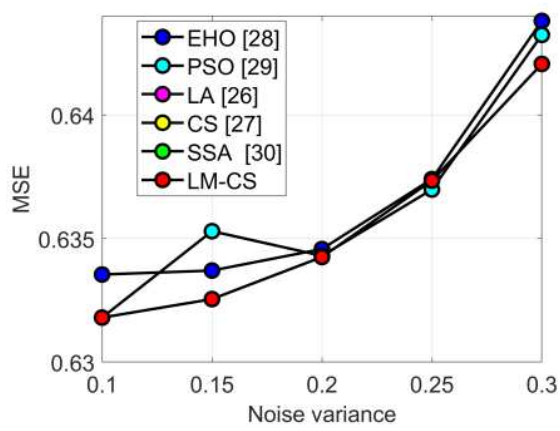

(b)

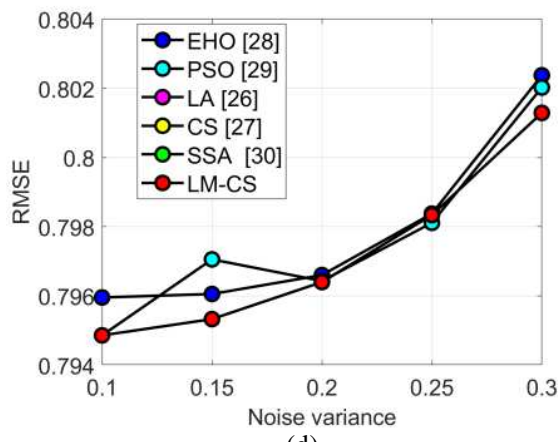

(d)

Fig. 6 Error Analysis of proposed model over state-of-the-art models by varying the noise (a) MAE (b) MSE (c) MAPE and (d) RMSE

\subsection{Convergence Analysis}

Fig. 7 expresses the convergence analysis of proposed model against the state-of-the-art models. In this section, the analysis is made regarding the cost function and the results are plotted. In order to make the system more effective, the cost function has to be attained minimum. It is observed that, the cost function of proposed model is initially at maximum that is in the range of 0.2982, which is gradually reduced as the iteration increases. More particularly, at the final iteration, the proposed model attains least convergence rate, which is below 0.2975 . At $20^{\text {th }}$ iteration, the introduced model is $0.012 \%$ and $0.59 \%$ improved than EHO and SSA, respectively with least cost function. From this analysis, it is clear that the performance of proposed model has attained better over other models with lower convergence rate. 


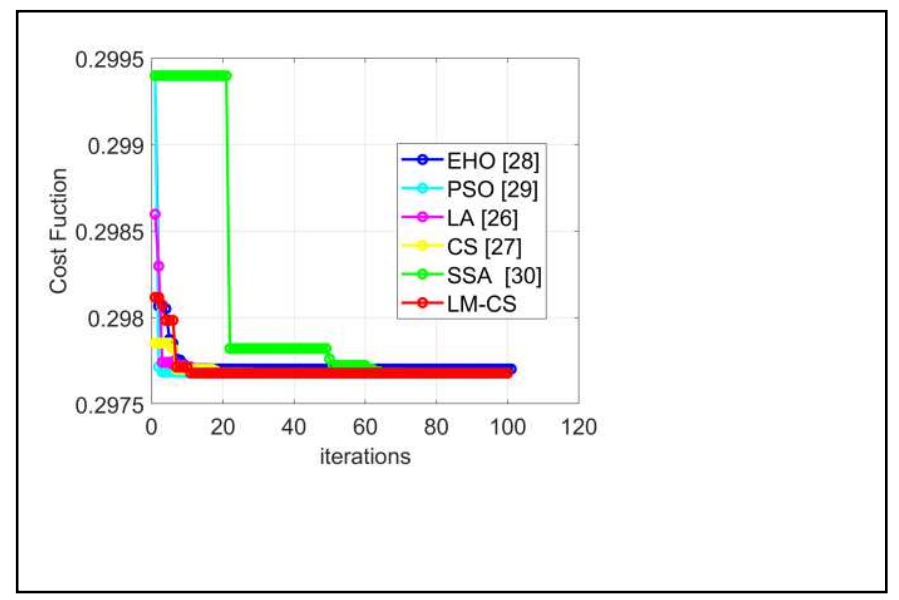

Fig. 7 Convergence Analysis of proposed model with state-of-the-art models

\subsection{Error Analysis by varying Reference Distance}

Fig. 8 delineates the error analysis of implemented model by varying the reference distance $d_{o}$. This analysis is made over the conventional models with varied reference distances to 1, 2, 3, 4 and 5. For MAE measure, the implemented model at reference distance 1 has achieved least error than other conventional models like EHO and SSA by $0.3 \%$ and $0.03 \%$, respectively. On considering MSE measure, the presented model is $0.12 \%$ and $0.02 \%$ superior to EHO and SSA, respectively for reference distance 2 with minimized error. In the view of MAPE at reference distance 3, the implemented approach is attained with least error performance, which is $0.09 \%, 0.1 \%$ and $0.57 \%$ better from EHO, PSO and SSA, respectively. Similarly for RMSE measure, the presented model is $0.04 \%$ and $0.006 \%$ improved than EHO and SSA with least error. The overall analysis thus proves the performance of proposed model with reduced error.

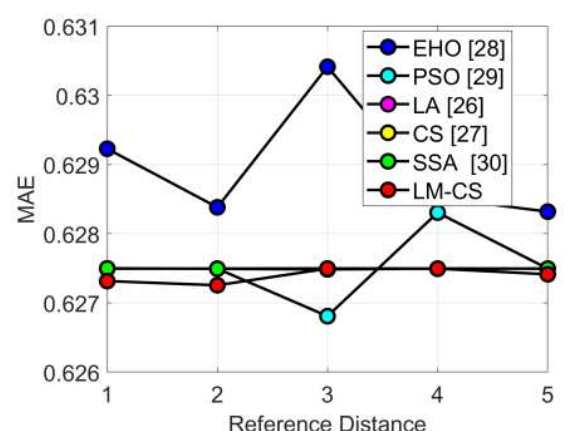

(a)

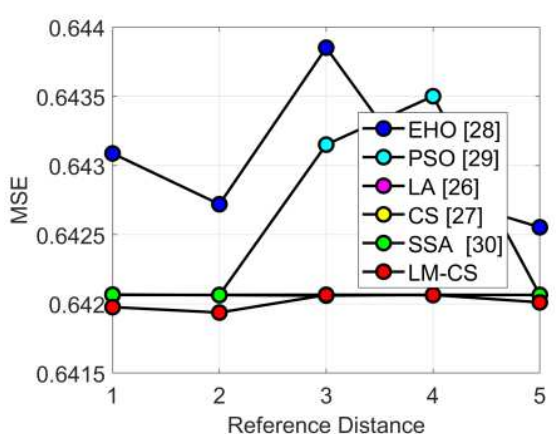

(b) 


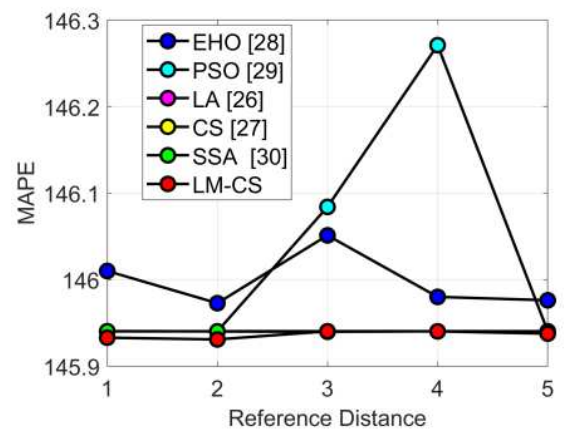

(c)

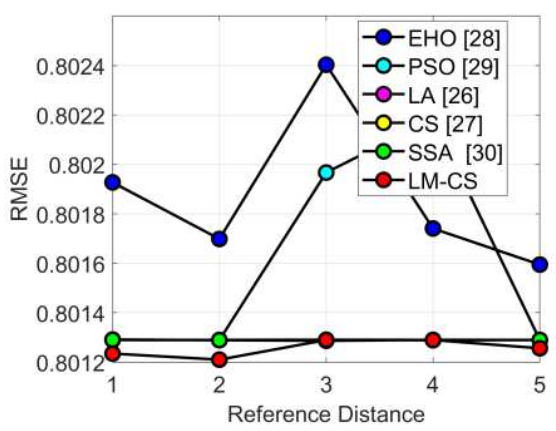

(d)

Fig. 8 Error Analysis of proposed model over state-of-the-art models by varying the reference distance (a) MAE (b) MSE (c) MAPE and (d) RMSE

\subsection{Error Analysis by varying the Static Sensor Count}

Fig. 9 portrays the error analysis of proposed model against the classical models by varying the number of static sensor. Here, the count of static sensor is varied as 2, 4, 6, 8 and 10. Initially, only two static sensors is taken and analyzed which results in maximized MAE error above 0.7 . Then the count of static sensors are increased gradually and analyzed. Hence, from the analysis, it is seemed that with the increase in the count of static sensor, the error gets minimized.

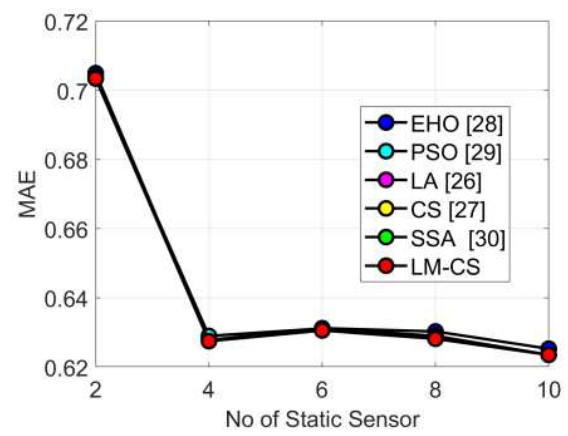

(a)

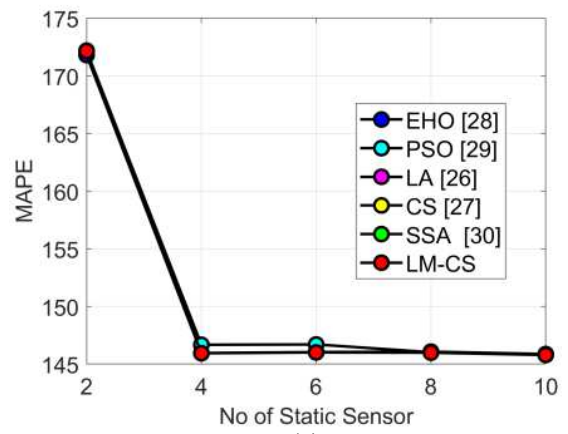

(c)

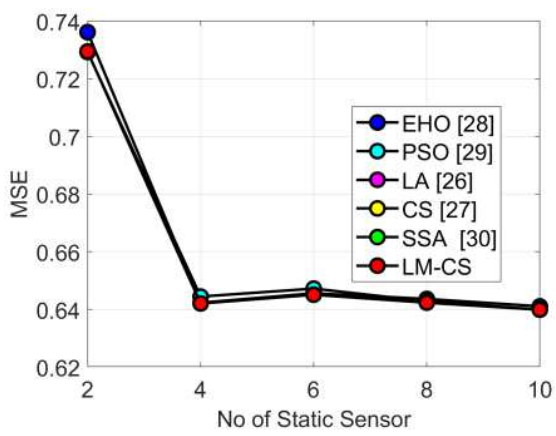

(b)

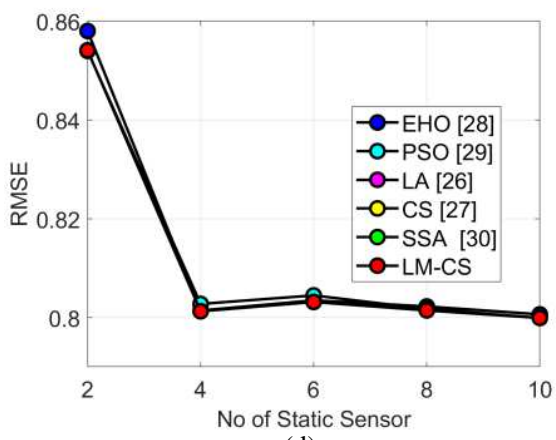

(d)

Fig. 9 Error Analysis of proposed model over state-of-the-art models by varying the count of static sensor (a) MAE (b) MSE (c) MAPE and (d) RMSE 


\subsection{Fitness Analysis}

Table 2, 3 and 4 explains the error analysis with respect to fitness function of proposed model by comparing other conventional models. The fitness function is evaluated based on $e_{1}$ and $e_{2}$ as in Eq. (12), which is the objective of this paper. The aim is to minimize $e_{1}$ and $e_{2}$. Table 2 summarizes the fitness analysis of proposed model against conventional models by varying the noise. The proposed model in terms of fitness $f$ at 0.3 noise variation is $0.02 \%$ and $0.14 \%$ superior to EHO and PSO, respectively. Table 3 explains the analysis on fitness of proposed model by varying the count of static sensor against traditional models. By fixing the static sensor as 2, the implemented model in terms of fitness function has gained betterment which is $1.03 \%, 0.33 \%, 0.017 \%, 0.001 \%$ and $0.65 \%$ improved than EHO, PSO, LA, CS and SSA, respectively. Table 4 describes the fitness analysis of proposed model against conventional models by varying the reference distance. The performance of implemented model at reference distance 3 achieves supremacy than EHO and SSA by $0.02 \%$ and $0.11 \%$, respectively. Thus the performance of the implemented model is validated by these results.

Table 2 Fitness Analysis of proposed model over state- of- the art models by varying the noise

\begin{tabular}{|c|c|c|c|c|c|c|c|c|c|}
\hline & \multicolumn{3}{|c|}{ EHO [28] } & \multicolumn{3}{|c|}{ PSO [29] } & \multicolumn{3}{|c|}{ LA [26] } \\
\hline $\begin{array}{r}\text { Noise } \\
\text { variation }\end{array}$ & $e_{1}$ & $e_{2}$ & $f$ & $e_{1}$ & $e_{2}$ & $f$ & $e_{1}$ & $e_{2}$ & $f$ \\
\hline 0.1 & 1.451282345 & 0.641810585 & 0.403422332 & 1.381227082 & 0.647506339 & 0.401322112 & 1.381227082 & 0.647506339 & 0.401322112 \\
\hline 0.15 & 1.407656651 & 0.641810585 & 0.402374285 & 1.348885566 & 0.647449628 & 0.40148131 & 1.34759431 & 0.647027194 & 0.401155351 \\
\hline 0.2 & 1.383954481 & 0.641810585 & 0.40521953 & 1.334567491 & 0.646387478 & 0.404967807 & 1.334520542 & 0.646422916 & 0.404965818 \\
\hline 0.25 & 1.379629821 & 0.641810585 & 0.410995411 & 1.338437212 & 0.645714555 & 0.410899184 & 1.338378903 & 0.645683995 & 0.410876733 \\
\hline \multirow[t]{2}{*}{0.3} & 1.392652023 & 0.641810585 & 0.417859878 & 1.358726885 & 0.645504754 & 0.418370945 & 1.356461648 & 0.644787915 & 0.417768379 \\
\hline & \multicolumn{3}{|c|}{ CS [27] } & \multicolumn{3}{|c|}{ SSA [30] } & \multicolumn{3}{|c|}{ LM-CS } \\
\hline $\begin{array}{r}\text { Noise } \\
\text { variation }\end{array}$ & $e_{1}$ & $e_{2}$ & $f$ & $e_{1}$ & $e_{2}$ & $f$ & $e_{1}$ & $e_{2}$ & $f$ \\
\hline 0.1 & 1.381227082 & 0.647506338 & 0.401322112 & 1.381227083 & 0.647506542 & 0.401322112 & 1.381227001 & 0.647506337 & 0.401322112 \\
\hline 0.15 & 1.347594313 & 0.64702719 & 0.401155351 & 1.34759431 & 0.647027194 & 0.401155352 & 1.347594328 & 0.647027064 & 0.401155351 \\
\hline 0.2 & 1.334520525 & 0.646422945 & 0.404965818 & 1.334520784 & 0.646422917 & 0.404965822 & 1.334520542 & 0.646422707 & 0.404965818 \\
\hline 0.25 & 1.338379026 & 0.645683846 & 0.410876732 & 1.338379025 & 0.645683916 & 0.410876732 & 1.338378964 & 0.645683846 & 0.410876732 \\
\hline 0.3 & 1.35646153 & 0.644788005 & 0.417768378 & 1.35646209 & 0.64478802 & 0.417768379 & 1.356461511 & 0.644787566 & 0.417768378 \\
\hline
\end{tabular}


Table 3 Fitness Analysis of proposed model over state- of- the art models by varying count of static sensor

\begin{tabular}{|c|c|c|c|c|c|c|c|c|c|}
\hline & \multicolumn{3}{|c|}{ EHO [28] } & \multicolumn{3}{|c|}{ PSO [29] } & \multicolumn{3}{|c|}{ LA [26] } \\
\hline $\begin{array}{r}\text { Count } \\
\text { of } \\
\text { static } \\
\text { sensor }\end{array}$ & $e_{1}$ & $e_{2}$ & $f$ & $e_{1}$ & $e_{2}$ & $f$ & $e_{1}$ & $e_{2}$ & $f$ \\
\hline 2 & 0.673790377 & 0.408783121 & 0.145557677 & 0.536813026 & 0.394275796 & 0.144539448 & 0.536294135 & 0.394309773 & 0.144083421 \\
\hline 4 & 1.392652023 & 0.641810585 & 0.417818058 & 1.357550169 & 0.645124144 & 0.418026146 & 1.356461883 & 0.644787729 & 0.417768379 \\
\hline 6 & 1.579799088 & 0.761785163 & 0.472693368 & 1.528121817 & 0.760377751 & 0.472742286 & 1.527078966 & 0.76017899 & 0.472541764 \\
\hline 8 & 2.015237887 & 0.902441175 & 0.590099945 & 1.973101542 & 0.904796765 & 0.590003213 & 1.973100957 & 0.904797327 & 0.590003214 \\
\hline 10 & 54790217 & 0.997423112 & 0.633660545 & 98682514 & 0.995853279 & 0.633213903 & 2.098682514 & 0.995853279 & 0.633213903 \\
\hline & \multicolumn{3}{|c|}{ CS [27] } & \multicolumn{3}{|c|}{ SSA [30] } & \multicolumn{3}{|c|}{ LM-CS } \\
\hline $\begin{array}{r}\text { Count } \\
\text { of } \\
\text { static } \\
\text { sensor }\end{array}$ & $e_{1}$ & $e_{2}$ & $f$ & $e_{1}$ & $e_{2}$ & $f$ & $e_{1}$ & $e_{2}$ & $f$ \\
\hline 2 & 0.536266121 & 0.394309451 & 14406111 & 0.537234883 & 0.394232933 & 0.145002125 & 0.536263993 & 0.394309158 & 0.144058983 \\
\hline 4 & 1.356461467 & 0.644788055 & 0.417768378 & 1.356461514 & 0.644788111 & 0.417768378 & 1.356461395 & 0.644788018 & 0.417768378 \\
\hline 6 & 1.527078728 & 0.760179251 & 0.472541759 & 1.527079335 & 0.760179269 & 0.472541883 & 1.527078713 & 0.760179079 & 0.47254176 \\
\hline 8 & 1.973101538 & 0.904796768 & 0.590003213 & 1.973101476 & 0.904995164 & 0.59002907 & 1.972992778 & 0.904796833 & 0.590003213 \\
\hline 10 & 2.098682505 & 0.995853291 & 0.633213903 & 2.098682687 & 0.995854273 & 0.633213905 & 2.098681787 & 0.995853073 & 0.633213903 \\
\hline
\end{tabular}

Table 4 Fitness Analysis of proposed model over state- of- the art models by varying reference distance

\begin{tabular}{|c|c|c|c|c|c|c|c|c|c|}
\hline & \multicolumn{3}{|r|}{ EHO [28] } & \multicolumn{3}{|r|}{ PSO [29] } & \multicolumn{3}{|r|}{ LA [26] } \\
\hline $\begin{array}{r}\text { Reference } \\
\text { distance }\end{array}$ & $e_{1}$ & $e_{2}$ & $f$ & $e_{1}$ & $e_{2}$ & $f$ & $e_{1}$ & $e_{2}$ & $f$ \\
\hline 1 & 1.392652023 & 0.641810585 & 0.417839833 & 1.35646151 & 0.644788021 & 0.417768378 & 1.356461412 & 0.644788106 & 0.417768381 \\
\hline 2 & 1.392652023 & 0.641810585 & 0.41784195 & 1.356461494 & 0.644788034 & 0.417768378 & 1.356461321 & 0.644788174 & 0.417768379 \\
\hline 3 & 1.392652023 & 0.641810585 & 0.417832687 & 1.35646151 & 0.644788021 & 0.417768378 & 1.356461294 & 0.64478819 & 0.417768379 \\
\hline 4 & 1.392652023 & 641810585 & 0.417850924 & 1.356972287 & 0.644957304 & 0.417899979 & 56461202 & 0.644788267 & 0.417768379 \\
\hline \multirow{2}{*}{\multicolumn{4}{|c|}{$\begin{array}{r}417829924 \\
\text { CS [27] } \\
\end{array}$}} & 1.356468484 & 0.644784428 & 0.417768909 & 1.356461758 & 0.64478783 & 0.417768378 \\
\hline & & & & \multicolumn{3}{|r|}{ SSA [30] } & \multicolumn{3}{|r|}{ LM-CS } \\
\hline $\begin{array}{r}\text { Reference } \\
\text { distance }\end{array}$ & $e_{1}$ & $e_{2}$ & $f$ & $e_{1}$ & $e_{2}$ & $f$ & $e_{1}$ & $e_{2}$ & $f$ \\
\hline 1 & 1.356461523 & 0.644788011 & 0.417768378 & 1.356432985 & 0.644815407 & 0.417769761 & 1.356462164 & 0.644787508 & 0.417768379 \\
\hline 2 & 56461555 & 0.644787986 & 0.417768378 & 1.356461084 & 0.644812723 & 0.417770231 & 43853 & 0.644788357 & 0.417768379 \\
\hline 3 & 6461798 & 644787795 & 0.417768379 & 9051 & 483533 & 8219974 & 59028 & 0.644789995 & 0.417768385 \\
\hline 4 & 1.356461409 & 0.644788101 & 0.417768379 & 1.35646183 & 0.644788021 & 0.417768379 & 1.35646151 & 0.644787772 & 0.417768378 \\
\hline 5 & 1.356461455 & 0.644788064 & 0.417768378 & 1.356461502 & 0.644788027 & 0.417768378 & 1.356461468 & 0.644788054 & 0.417768378 \\
\hline
\end{tabular}

\subsection{Statistical Analysis}

Table 5 expresses the statistical analysis of implemented model over conventional models. When differentiating the implemented from conventional model, the proposed model has attained better results with least error which results in improved performance. For MAE, the adopted approach in terms of best case scenario is $0.18 \%, 0.07 \%, 0.04 \%, 0.04 \%$ and $0.037 \%$, respectively. Similarly, on considering MSE for mean performance, the implemented model is $0.17 \%$ and $0.11 \%$ better from $\mathrm{EHO}$ and $\mathrm{PSO}$, and $0.08 \%$ better from other models like LA, CS and SSA. The performance is thus compared and analyzed for other error measure and has thus attained the minimum error, thereby validates the improved performance of proposed model. 
Table 5 Statistical Analysis of proposed model against conventional models

\begin{tabular}{|c|r|r|r|r|r|}
\hline \multicolumn{5}{|c|}{ MAE } \\
\hline Methods & Best & Worst & Mean & Median & Std deviation \\
\hline EHO [28] & 0.628381 & 0.6304129 & 0.6291360 & 0.628875 & 0.00092831 \\
\hline PSO [29] & 0.626808 & 0.6283047 & 0.6275257 & 0.6274951 & 0.00061212 \\
\hline LA [26] & 0.627493 & 0.6274993 & 0.6274953 & 0.6274944 & $2.99 \mathrm{e}^{-06}$ \\
\hline CS [27] & 0.6274954 & 0.6274974 & 0.627496 & 0.6274956 & $9.55 \mathrm{e}^{-07}$ \\
\hline SSA [30] & 0.6274842 & 0.627498 & 0.6274931 & 0.6274950 & $6.26 \mathrm{e}^{-06}$ \\
\hline LM-CS & 0.6272549 & 0.6274952 & 0.6273901 & 0.6274051 & 0.00012382 \\
\hline \multicolumn{7}{|c|}{ MSE } \\
\hline Methods & Best & Worst & Mean & Median & Std deviation \\
\hline EHO [28] & 0.6427201 & 0.6438512 & 0.6431117 & 0.6429376 & 0.00051818 \\
\hline PSO [29] & 0.6420645 & 0.6435007 & 0.6426952 & 0.6426078 & 0.00074206 \\
\hline LA [26] & 0.6420632 & 0.6420674 & 0.6420647 & 0.6420642 & $2.00 \mathrm{e}^{-06}$ \\
\hline CS [27] & 0.6420647 & 0.6420659 & 0.6420651 & 0.6420649 & $5.43 \mathrm{e}^{-07}$ \\
\hline SSA [30] & 0.6420597 & 0.6420659 & 0.6420638 & 0.6420647 & $2.85 \mathrm{e}^{-06}$ \\
\hline LM-CS & 0.6419373 & 0.6420646 & 0.6420107 & 0.6420206 & $6.42 \mathrm{e}^{-05}$ \\
\hline \multicolumn{7}{|c|}{ MAPE } \\
\hline Methods & Best & Worst & Mean & Median & Std deviation \\
\hline EHO [28] & 145.97272 & 146.05113 & 146.00345 & 145.99497 & 0.03563935 \\
\hline PSO [29] & 145.94019 & 146.27107 & 146.05888 & 146.01213 & 0.1568795 \\
\hline LA [26] & 145.94012 & 145.94033 & 145.94019 & 145.94017 & $9.53 \mathrm{e}^{-05}$ \\
\hline CS [27] & 145.94021 & 145.94028 & 145.94023 & 145.94021 & $3.68 \mathrm{e}^{-05}$ \\
\hline SSA [30] & 145.9397 & 145.94033 & 145.94009 & 145.94018 & 0.00028127 \\
\hline LM-CS & 145.93081 & 145.94019 & 145.93600 & 145.93649 & 0.0049124 \\
\hline \multicolumn{7}{|c|}{ RMSE } \\
\hline Methods & Best & Worst & Mean & Median & Std deviation $^{|c|}$ \\
\hline EHO [28] & 0.8016983 & 0.8024034 & 0.8019424 & 0.8018339 & 0.00032303 \\
\hline PSO [29] & 0.8012892 & 0.8021849 & 0.8016826 & 0.801628 & 0.00046279 \\
\hline LA [26] & 0.8012885 & 0.8012911 & 0.8012894 & 0.8012891 & $1.25 \mathrm{e}^{-06}$ \\
\hline CS [27] & 0.8012894 & 0.8012902 & 0.8012897 & 0.8012895 & $3.39 \mathrm{e}^{-07}$ \\
\hline SSA [30] & 0.8012863 & 0.8012902 & 0.8012888 & 0.8012894 & $1.78 \mathrm{e}^{-06}$ \\
\hline LM-CS & 0.8012099 & 0.8012894 & 0.8012557 & 0.8012619 & $4.01 \mathrm{e}^{-05}$ \\
\hline
\end{tabular}

\section{Conclusion}

This work has introduced a new energy-saving target tracking scheme that consist of two main phases: (i) Mobility Target Tracking and (ii) Target Movement Prediction. Firstly, the Extended Kalman Filter was deployed for attaining the target tracking. The target movement prediction was pursued with the help of input factors such as AoA and RSS, which in turns predict the mobile node's optimal movement. In order to make the optimal prediction of target movement, this paper presented a novel hybrid optimization algorithm named LM-CS, which was the hybridized concept of LA and CS. The evaluation on performance was performed via various analyses and thus validated the dominance of the implemented model. While analysing MAE, the implemented model with noise variance 0.15 has achieved better results with least error, which was $0.45 \%$ and $0.3 \%$ improved than EHO and PSO, respectively. Similarly, for noise variance 0.3 , the presented model was $0.32 \%$ and $0.27 \%$ superior to EHO and PSO, respectively. On considering the MSE measure, the proposed 
model in noise variance 0.3 gained supremacy than conventional models EHO and PSO by $0.18 \%$ and $0.07 \%$, respectively with minimum error.

\section{Declarations}

Funding Not Applicable.

Conflicts of interest/Competing interests Not Applicable.

Availability of Data and Material All data generated or analysed during this study are included in this published article( and its supplementary information files).

Code availability The custom code will be shared whenever it is required for the review. Authors' contribution All the authors contributed their skills and efforts to complete this study and produce the final article.

\section{References}

1. ParulpreetSingh, ArunKhosla, AnilKumar, MamtaKhosla," Optimized localization of target nodes using single mobile anchor node in wireless sensor network", AEU International Journal of Electronics and Communications, vol.91, pp.55-65, July 2018. https://doi.org/10.1016/j.aeue.2018.04.024

2. BaomingSun, YanGuo, NingLi, LaixianPeng, DagangFang," TDL: Two-dimensional localization for mobile targets using compressive sensing in wireless sensor networks", Computer Communications, vol.78, pp.45-55, 15 March 2016.

3. SaharKouroshnezhad, AliPeiravi, MohammadSayad Haghighi, QiZhang," A mixedinteger linear programming approach for energy-constrained mobile anchor path planning in wireless sensor networks localization", Ad Hoc Networks, vol.87, pp.188-199, 1 May 2019.

4. SupengSun, JianpingZhao, XiaochuanTian, JingZhang," Path planning for multiple mobile anchor nodes assisted localization in wireless sensor networks", Measurement, vol.141, pp.124-136, July 2019.

5. C. Kuo, T. Chen and S. Syu, "Robust Mechanism of Trap Coverage and Target Tracking in Mobile Sensor Networks," IEEE Internet of Things Journal, vol. 5, no. 4, pp. 30193030, Aug. 2018. 
6. Tao Wang, Xiang Wang, Wei Shi, Zongmin Zhao, Tongsheng Xia," Target Localization and Tracking Based on Improved Bayesian Enhanced Least-Squares Algorithm in Wireless Sensor Networks", Computer Networks, In press, journal pre-proof, Available online 5 November 2019.

7. Charly Lersteau, André Rossi, Marc Sevaux," Minimum energy target tracking with coverage guarantee in wireless sensor networks", European Journal of Operational Research, vol.265, no.3, pp.882-894, 16 March 2018.

8. Amir Javadpour," An Optimize-Aware Target Tracking Method Combining MAC Layer and Active Nodes in Wireless Sensor Networks", Wireless Personal Communications, pp 1-18, 25 April 2019.

9. XiuwenFu, GiancarloFortino, WenfengLi, PasqualePace, YongshengYang," WSNsassisted opportunistic network for low-latency message forwarding in sparse settings", Future Generation Computer Systems, vol.91, pp.223-237, February 2019.

10. V. Jyothi,M.V. Subramanyam, "An Enhanced technique to improve the Network Life time of Cognitive Sensor Networks, Wireless Personal Communications, pp. 12757 - 12763, May-2021. https://doi.org/10.1007/s11277-021-08575-0

11. Trupti M Behera, Sushanta K Mohapatra, Umesh C Samal, Mohammad S Khan, "Hybrid heterogeneous routing scheme for improved network performance in WSNs for animal tracking", Internet of Things, vol.6, June 2019. https://doi.org/10.1016/j.iot.2019.03.001

12. Ruhul Amin, SK Hafizul Islam, G. P. Biswas, Mohammad S. Obaidat," A robust mutual authentication protocol for WSN with multiple base-stations", Ad Hoc Networks, vol.75768, pp.1-18, June 2018.

13. Ian T. Ruginski, Sarah H. Creem-Regehr, Jeanine K. Stefanucci, Elizabeth Cashdan," GPS use negatively affects environmental learning through spatial transformation abilities", Journal of Environmental Psychology, vol.64, pp.12-20, August 2019

14. M. Durand, A. Rivera, F. Geremia-Nievinski, M. G. Lenzano, L. Lenzano," GPS reflectometry study detecting snow height changes in the Southern Patagonia Icefield", Cold Regions Science and Technology, vol.166, October 2019.

15. Lucy Joseph, An Neven, Karel Martens, Opportuna Kweka, Davy Janssens," Measuring individuals' travel behaviour by use of a GPS-based smartphone application in Dar es 
Salaam, Tanzania", Journal of Transport Geography, In press, corrected proof, Available online 18 July 2019.

16. Tarek R. Sheltami, Essa Q. Shahra, Elhadi M. Shakshuki," Perfomance comparison of three localization protocols in WSN using Cooja", Journal of Ambient Intelligence and Humanized Computing, vol.8, no3, pp 373-382, June 2017.

17. Md Al Shayokh, Soo Young Shin," Bio Inspired Distributed WSN Localization Based on Chicken Swarm Optimization", Wireless Personal Communications, vol.97, no.4, pp 5691-5706, December 2017.

18. WenJie Wang, BoBin Yao, QinYe Yin," AOD estimation in WSN localization system with synthetic aperture technique", Science China Information Sciences, vol.55, no.10, pp 2216-2225, October 2012.

19. Sheng Feng, Chengdong Wu, Yunzhou Zhang, Gabriele Oliva," WSN Deployment and Localization Using a Mobile Agent", Wireless Personal Communications, vol.97, no.4, pp 4921-4931, December 2017.

20. Stephan Schlupkothen, Bastian Prasse, Gerd Ascheid," Backtracking-based dynamic programming for resolving transmit ambiguities in WSN localization", EURASIP Journal on Advances in Signal Processing, December 2018.

21. Mohammad Gholami, Robert W. Brennan," Comparing two clustering-based techniques to track mobile nodes in industrial wireless sensor networks", Journal of Systems Science and Systems Engineering, vol.25, no.2, pp 177-209, June 2016.

22. Yi Yang, Lian Li, Hao Li," Data forwarding of realtime mobile target tracking in wireless sensor networks", Journal of Ambient Intelligence and Humanized Computing, vol.4, no.1, pp 109-120, February 2013.

23. Tongxiang Wang, Xianglin Wei, Fei Hu, Jianhua Fan," Mobile jammer localization and tracking in multi-hop wireless network", Journal of Ambient Intelligence and Humanized Computing, pp 1-12, 20 February 2018.

24. Wei Chen, Yinfei Fu," Cooperative distributed target tracking algorithm in mobile wireless sensor networks", Journal of Control Theory and Applications, May 2011.

25. Bartłomiej Płaczek," Decision-aware data suppression in wireless sensor networks for target tracking applications", Frontiers of Computer Science, vol.11, no.6, pp 1050-1060, December 2017. 
26. Rajakumar Boothalingam, " Optimization using lion algorithm: a biological inspiration from lion's social behavior", Evolutionary Intelligence, vol.11, no. 1-2, pp.31-52, 2018.

27. M. Mareli and B. Twala," An adaptive Cuckoo search algorithm for optimisation," Applied Soft Computing, vol. 37, pp.332-344, December 2015.

28. Wang, Gai-Ge \& Deb, Suash \& Coelho, Leandro," Elephant Herding Optimization", 2015.

29. M.E.H.Pedersen and A.J.Chipperfield, " Simplifying Particle Swarm Optimization", Applied Soft Computing, vol. 10, no. 2, pp. 618-628, 2010.

30. SeyedaliMirjalili, Amir H.Gandomi, Seyedeh ZahraMirjalili, ShahrzadSaremi, HossamFaris, Seyed MohammadMirjalili," Salp Swarm Algorithm: A bio-inspired optimizer for engineering design problems", Advances in Engineering Software, vol.114, pp.163-191, December 2017.

31. Y.Mallikarjuna Rao, M.V.Subramanyam and K. Satyaprasad, "Cluster based hybrid routing protocol for wireless mesh networks", Wireless personal communications, Vol.103,no.4,pp.3009-3023,2018. 


\section{Supplementary Files}

This is a list of supplementary files associated with this preprint. Click to download.

- Datageneratedandanalysedsupplementaryfile.xlsx 